\title{
Screening for pollen tolerance to high temperatures in tomato
}

\author{
Marine J. Paupière · Pauline van Haperen · Ivo Rieu • Richard G. F. Visser • \\ Yury M. Tikunov $\cdot$ Arnaud G. Bovy
}

Received: 3 April 2017/ Accepted: 6 June 2017/Published online: 10 June 2017

(C) The Author(s) 2017. This article is an open access publication

\begin{abstract}
Among the abiotic stresses affecting plant reproduction, high temperature is one of the most prominent ones because it directly affects fruit set. So far, little attention has been paid to the investigation of the variation in high temperature tolerance among wild tomato (Solanum lycopersicum) germplasm. The objective of this study was to determine the tolerance of 17 different cultivated and wild tomato accessions to high temperature, using a pollen viability screening approach. Each of the 17 genotypes of tomato was analysed for their pollen quality under a $32{ }^{\circ} \mathrm{C}$ (day)/ $26{ }^{\circ} \mathrm{C}$ (night) regime. The total number of pollen per flower and the fraction of viable pollen were recorded. The number of pollen per flower varied between 35,547 and 109,490 whereas the fraction of viable pollen varied between 0.03 and 0.71 . No correlation was found between these two traits. However, the combination of these traits could provide the best reproductive capability under high temperature. In this study, thermo-tolerant (LA2854, LA1478 and
\end{abstract}

M. J. Paupière · P. van Haperen · R. G. F. Visser ·

Y. M. Tikunov · A. G. Bovy $(\square)$

Plant Breeding, Wageningen University \& Research,

Droevendaalsesteeg 1, 6708PB Wageningen, The

Netherlands

e-mail: arnaud.bovy@wur.nl

I. Rieu

Molecular Plant Physiology, Institute for Water and Wetland Research, Radboud University, Heyendaalseweg 135, 6525AJ Nijmegen, The Netherlands
LA0417) as well as thermo-sensitive (LA1719, LA1580, and SWEET4) genotypes have been identified. Those genotypes can be used as novel genetic resources to get more insight into pollen thermotolerance mechanisms and be included in breeding programs.

Keywords Pollen viability $\cdot$ Heat stress $\cdot$ High temperature $\cdot$ Tomato $\cdot$ Screening $\cdot$ Breeding .

Tolerance

\section{Introduction}

High temperature is one of the major abiotic stresses affecting plant reproduction, and therefore, fruit set (Dane et al. 1991). Most commercial tomato (Solanum lycopersicum) genotypes are not tolerant to high temperatures. A rise of a few degrees above the optimum growing temperature will, consequently, lead to a decrease of fruit set. Optimum growing temperatures for tomato are between 18 and $25{ }^{\circ} \mathrm{C}$ (Hurd and Cooper 1970). The predicted increase of $1-3{ }^{\circ} \mathrm{C}$ during the twenty-first century (IPCC 2012) may lead to a decrease of tomato production. Developing tomato genotypes tolerant to high temperature may be a valuable strategy to cope with these environmental changes. Tolerance to high temperature is not an easy trait to improve due to its low heritability (Hanson et al. 2002; Hazra et al. 2009), possibly due to its sensitivity to other environmental factors such as humidity 
(Abdulbaki 1991). To determine the tolerance of a plant to high temperature, different parameters (Wahid et al. 2007) can be recorded, such as cell membrane thermostability, photosynthetic activity, pollen viability and fruit set. Fruit set is the ultimate measure for the tolerance of a genotype to high temperature and has been shown to correlate with a decrease of pollen viability in tomato (Firon et al. 2006). In general, genotypes tolerant to high temperature maintain a higher level of pollen viability under high temperatures than sensitive genotypes (Dane et al. 1991). The use of pollen viability as a screening tool will provide valuable information about the male gametophytic tolerance of different tomato genotypes to high temperatures. It is expected that focus on one aspect of the heat tolerance mechanism, pollen, will have simpler genetics, compared to screening for fruit production, which is a much more complex trait, determined by many more factors, such as flower formation, male and female tolerance, fruit set and fruit development. This is much more difficult to dissect genetically.

Several studies have focused on the identification of tomato cultivars tolerant to high temperature (Abdulbaki 1991; Abdelmageed and Gruda 2009; Da Costa et al. 2011; Kartikeya et al. 2012; Kugblenu et al. 2013). However, due to domestication and intensive breeding, the cultivated tomato germplasm has a rather narrow genetic basis. In contrast, there is a much larger genetic diversity in related wild relatives of tomato (Viquez-Zamora et al. 2013) and, hence, these may form an alternative source of thermo-tolerant genotypes. To date, little attention has been paid to the investigation of the variation in high temperature tolerance among the wild tomato germplasm. The identification of tolerant wild accessions may provide an additional valuable resource to develop tolerant tomato genotypes through classical breeding.

The objective of this study was to determine the tolerance of different cultivated and wild tomato accessions to high temperature, using a pollen viability screening approach.

\section{Materials and methods}

Plant materials

Seventeen different tomato genotypes were tested under heat stress conditions in this study. Six wild
S. pimpinellifolium accessions: LA0417, LA1478, LA1719, LA1580, LA1584 and LA2854 were obtained from the Tomato Genetics Resource Centre (TGRC). These accessions were selected based on the environmental temperature and the altitude of the geographic locations they were collected from (Fig. 1). All the S. pimpinellifolium accessions originated from locations with low altitude and hot environmental conditions-maximum average day temperature $28-31{ }^{\circ} \mathrm{C}$. Seven $S$. lycopersicum genotypes: CLN1621F, CL5915-93D4-1-0-3, CL1131-00-13-0-6, CLN475BC1F2-265-4-19, CLN65-349D52-0, CL5915-206D4-2-2-0-4 and CL5915-153D4-3-30 were obtained from the Asian Vegetable Research and Development Centre (AVRDC) where they were annotated as 'tolerant to heat stress'. Solanum lycopersicum varieties Saladette and Nagcarlang were obtained from Radboud University, Nijmegen, The Netherlands and were annotated 'tolerant to heat stress' in the database of TGRC. SWEET4 is an introgression line developed at Wageningen UR plant breeding, The Netherlands, from a cross between $S$. lycopersicum cv. Moneymaker and S. chmielewskii LA1028. This introgression line was selected for its general vigour. Solanum lycopersicum variety M-82 obtained from TGRC was used as thermo-sensitive control.

Experimental growth conditions

The experiment was conducted in two periods. In the first period (March 2013), eight genotypes (CLN1621F, CL5915-93D4-1-0-3, CL1131-0-0-130-6, CLN475BC1F2-265-4-19, CLN65-349D5-2-0, CL5915-206D4-2-2-0-4, CL5915-153D4-3-3-0 and M-82) were used and in the second period (April 2013), nine additional genotypes were used (LA2854, LA1478, LA0417, LA1584, LA1580, LA1719, Nagcarlang, Saladette and SWEET4). Plants were grown in the greenhouse of Unifarm (Wageningen University $\&$ Research Centre, The Netherlands) at $25{ }^{\circ} \mathrm{C}$ day and $19{ }^{\circ} \mathrm{C}$ night under $12-18 \mathrm{~h}$ of natural day light for 1 month (control condition). When the first flowers appeared, plants were moved and placed into a $15 \mathrm{~m}^{2}$ climate chamber using a randomized design. The climate chamber was conditioned at $32 / 26{ }^{\circ} \mathrm{C}$, with $12 \mathrm{~h}$ of day light provided with fluorescent tubes at a photon flux density of $500 \mu \mathrm{mol}$ and $60 \%$ humidity. All flowers and buds from each plant were removed 
Fig. 1 Geographical origins of $S$. pimpinellifolium accessions. $\mathrm{X}, \mathrm{X}, \mathrm{X}, \mathrm{X}$ represents accession number from TGRC, site of collection, metres above sea level, average temperature. ${ }^{l}$ Data temperature of Guayaquil, Ecuador. ${ }^{2}$ Data temperature of Chulucanas, Peru (http:// en.climate-data.org/)

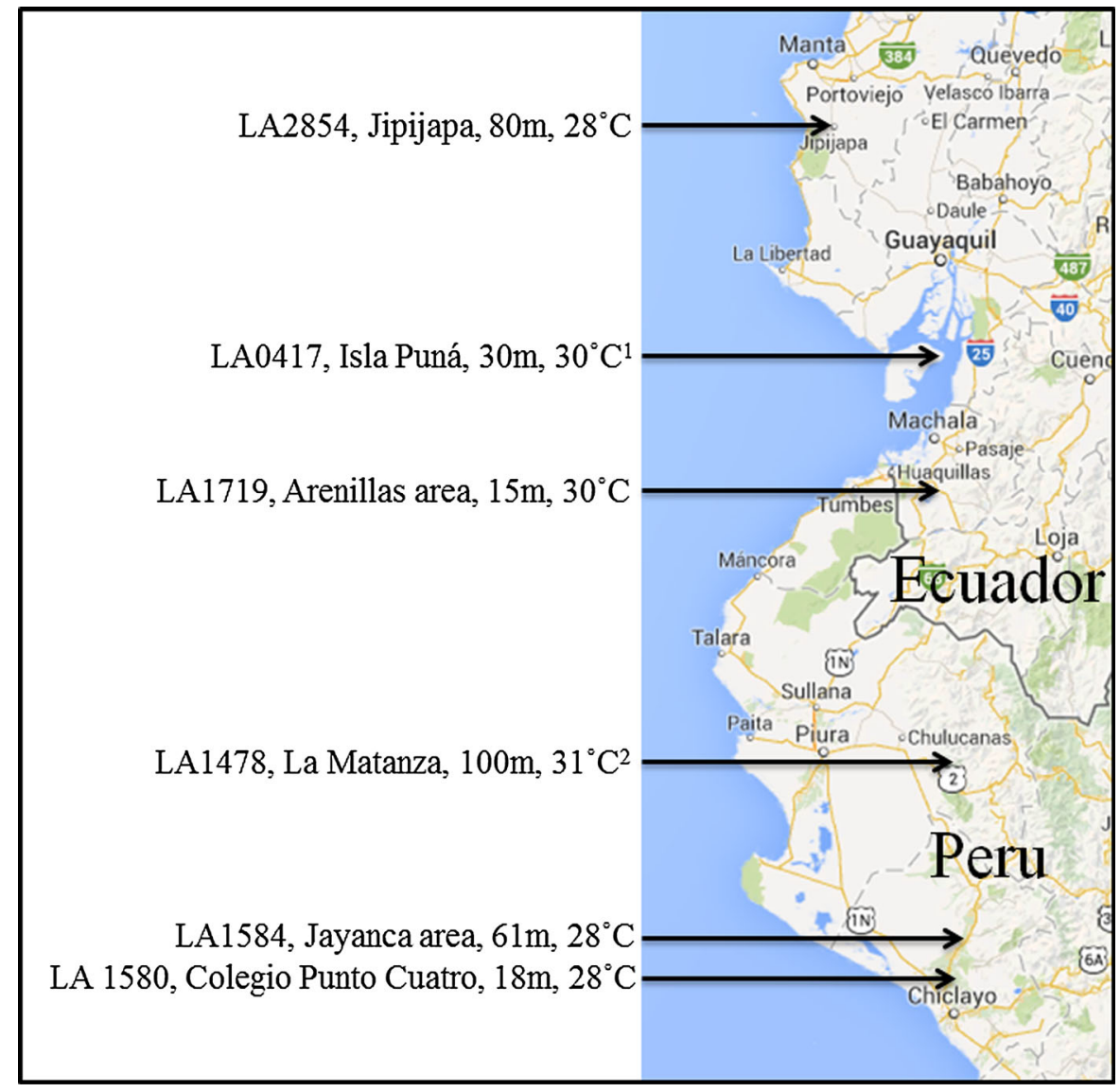

with forceps to assure that new flowers have been completely developed under the climate chamber conditions. 2 weeks were needed to produce new open flowers.

\section{Pollen viability test}

For the pollen viability screening analysis, newly opened flowers were collected each morning from 9 to 11 a.m. under the heat stress. In total, ten flowers per plant were collected and six plants were used per genotype. Flowers were collected into a petri dish filled with a wet paper. From each flower, anthers were cut into three to four pieces with a razor blade on a glass and put in an Eppendorf tube of $1.5 \mathrm{~mL}$. Then $0.5 \mathrm{~mL}$ of germination solution consisting of $1 \mathrm{mM}$ $\mathrm{KNO}_{3}, 3 \mathrm{mM} \mathrm{Ca}\left(\mathrm{NO}_{3}\right) 2 \cdot 4 \mathrm{H}_{2} \mathrm{O}, 0.8 \mathrm{mM} \mathrm{MgSO}{ }_{4} \cdot 7$ $\mathrm{H}_{2} \mathrm{O}, 1.6 \mathrm{mM} \mathrm{H}_{3} \mathrm{BO}_{3}$ was added to the Eppendorf tube [adapted from Pressman et al. (2002)], followed by 20 $\mu \mathrm{L}$ of Alexander dye. The Alexander dye consisted of
$10 \mathrm{~mL}$ of $95 \%$ alcohol, $1 \mathrm{~mL}$ of malachite green $(1 \%$ solution in $95 \%$ alcohol), $54.5 \mathrm{~mL}$ of distilled water, $25 \mathrm{~mL}$ of glycerol, $5 \mathrm{~mL}$ of Acid fuchsin (1\% solution in water), $0.5 \mathrm{~mL}$ of Orange $\mathrm{G}$ ( $1 \%$ solution in water) and $4 \mathrm{~mL}$ of glacial acetic acid for a $100 \mathrm{~mL}$ solution (Alexander 1980; Peterson et al. 2010). The Eppendorf tube was then vortexed for $10 \mathrm{~s}$ and kept overnight at room temperature. The day after, pollen number was counted using a Fuchs-Rosenthal haemocytometer (W. Schreck Hofheim/Ts). $10 \mu \mathrm{L}$ of pollen solution was loaded into the haemocytometer and the number of viable pollen (stained purple) and non-viable pollen (stained green) were counted in eight squares of the haemocytometer for each sample using a light microscope. The counted number of pollen was then transformed in number of pollen per flower using the following formula:

Number of pollen per flower

$=$ Number of pollen per square $* 2500^{\mathrm{a}}$ 
a was calculated based on the fact that each square contained $0.2 \mu \mathrm{L}$ of pollen solution, and the pollen of one flower was isolated in $500 \mu \mathrm{L}$. Hence to determine the number of pollen per flower, the number of pollen per square was multiplied by 2500 .

Pollen number counting for the first part of the experiment was performed in May 2013 whereas pollen number counting for the second part of the experiment was performed in July 2013.

\section{Statistical analysis}

Six biological replicates were used for each of the genotypes tested except for LA1478 for which four biological replicates were used. Each replicate represented a pool of pollen derived from ten flowers from the same plant. The fraction of viable pollen was determined by dividing the number of viable pollen by the total number of pollen. Subsequently, an ANOVA test was performed together with a Tukey test. Genotypes from different growing periods were analysed independently. All analyses were done using SPSS 20ed.

\section{Results}

Genotypic variation in total pollen production under heat stress

The total number of pollen produced under the high temperature $\left(32 / 26{ }^{\circ} \mathrm{C}\right)$ varied between the 17 accessions tested (Fig. 2). Among the genotypes of the first period, the highest pollen number per flower was 109,490 (CLN1621F) and the lowest pollen number per flower was 60,266 (CL1131-0-0-13-0-6) under high temperature. The genotype CLN1621F showed significantly more pollen number per flower than the genotypes CL1131-0-0-13-0-6, CLN475BC1F2-2654-19, CL5915-153D4-3-3-0. Among the genotypes of the second period, the highest pollen number per flower was 96,157 (LA1580) and the lowest pollen number per flower was 35,547 (LA1719) under high temperature. The genotype LA1580 showed significantly higher pollen number per flower than the genotypes LA1719, SWEET4 and Saladette. For instance, LA1580 produced two times more pollen than Saladette under high temperatures.
Genotypic variation in pollen viability under heat stress

The fraction of viable pollen under the high temperature $\left(32 / 26{ }^{\circ} \mathrm{C}\right)$ varied between the 17 accessions tested (Fig. 3). Among the genotypes of the first period, the highest ratio of viable pollen was 0.58 (CL5915-153D4-3-3-0) and the lowest ratio of viable pollen was 0.28 (M-82) under high temperature. The genotypes CL5915-153D4-3-3-0, CL1131-0-0-13-06, CLN1621F and CL5915-93D4-1-0-3 had a significantly higher ratio of viable pollen than M-82. In general, all seven genotypes obtained from AVRDC showed no statistically significant difference in pollen viability, which varied within $10 \%$ between them. More variation in pollen viability was observed in the second growing period. The highest ratio of viable pollen under the heat condition was 0.71 (LA2854) and the lowest ratio of viable pollen was 0.03 (SWEET4). The genotypes LA2854, Nagcarlang and LA1478 showed a significantly higher ratio of viable pollen than SWEET4, LA1719 and LA1580 under high temperature. There was no significant correlation observed between the total number of pollen and the viable fraction of pollen produced under high temperature (Fig. 4). The Pearson correlation between these two traits is 0.19 .

Based on their response to the two parameters tested, the genotypes can be considered at four different levels, with genotypes producing (i) a high total number of pollen with a high fraction of viable pollen (e.g. LA2854 and CLN1621F), (ii) a high total number of pollen with a low fraction of viable pollen (e.g. LA1580), (iii) a low total number of pollen with a high fraction of viable pollen (e.g. Saladette), (iv) a low total number of pollen with a low fraction of viable pollen (e.g. SWEET4).

\section{Discussion}

Wild tomato germplasm has been successfully used in breeding programs to introduce different traits, such as various disease resistance genes, into cultivated tomato (Labate and Robertson 2012). In this study, we have selected six $S$. pimpinellifolium accessions, which originated from geographical regions with high average temperatures. All the selected $S$. pimpinellifolium accessions originated from a similar 


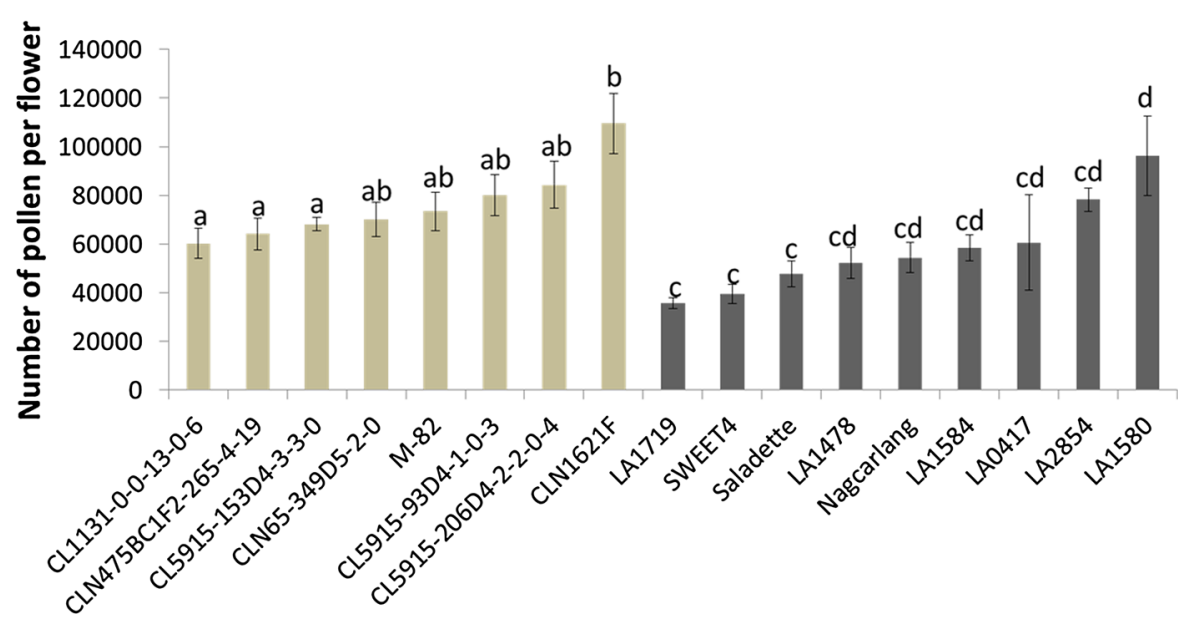

Fig. 2 Number of pollen produced per flower per genotype under heat stress $\left(32 / 26^{\circ} \mathrm{C}\right)$. Light bars represent genotypes grown in the first period whereas dark bars represent genotypes grown in the second period. Error bars represent standard error of the biological replicate mean. The letter above the bar represents the statistical difference based on a one way ANOVA with a Tukey's post hoc test for the number of pollen per flower and per period. Statistics were performed independently for both periods. Genotypes with the same letter did not show statistically significant differences between each other $(p$ value $>0.05)$

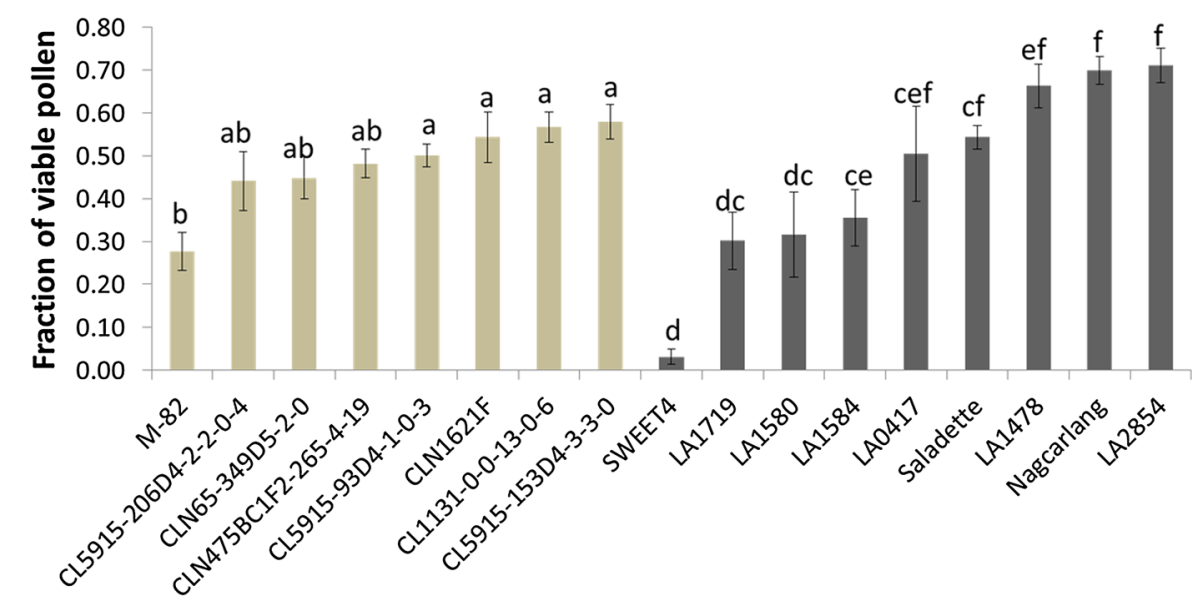

Fig. 3 Fraction of viable pollen per genotype under heat stress $\left(32 / 26^{\circ} \mathrm{C}\right)$. Light bars represent genotypes grown in the first period whereas dark bars represent genotypes grown in the second period. Error bars represent standard error of the biological replicate mean. The letter above the bar represents

environment in terms of average maximum temperature ranging from 28 to $31^{\circ} \mathrm{C}$, which is higher than the optimal growing temperature for cultivated tomato (which is between 18 and $25^{\circ} \mathrm{C}$ ). These accessions were tested for total pollen number and pollen viability under high temperatures $\left(32{ }^{\circ} \mathrm{C}\right.$ during the day and $26{ }^{\circ} \mathrm{C}$ during the night) together with nine tomato genotypes, which have been reported to be tolerant to high temperature. Despite the similarity in their the statistical difference based on a one way ANOVA with a Tukey's post hoc test for the fraction of viable pollen. Statistics were performed independently for both periods. Genotypes with the same letter did not have statistically significant differences between each other ( $\mathrm{p}$ value $>0.05$ )

original growing environment, the $S$. pimpinellifolium accessions showed a high variation in both the total number of pollen and the fraction of viable pollen produced under high temperature. Among these six genotypes, three had a viable pollen fraction higher than 0.5 . LA2854 and LA1478 belonged to the group with the highest fraction of viable pollen whereas LA1719 and LA1580 did not differ significantly from SWEET4 which had a pollen viability close to zero. 


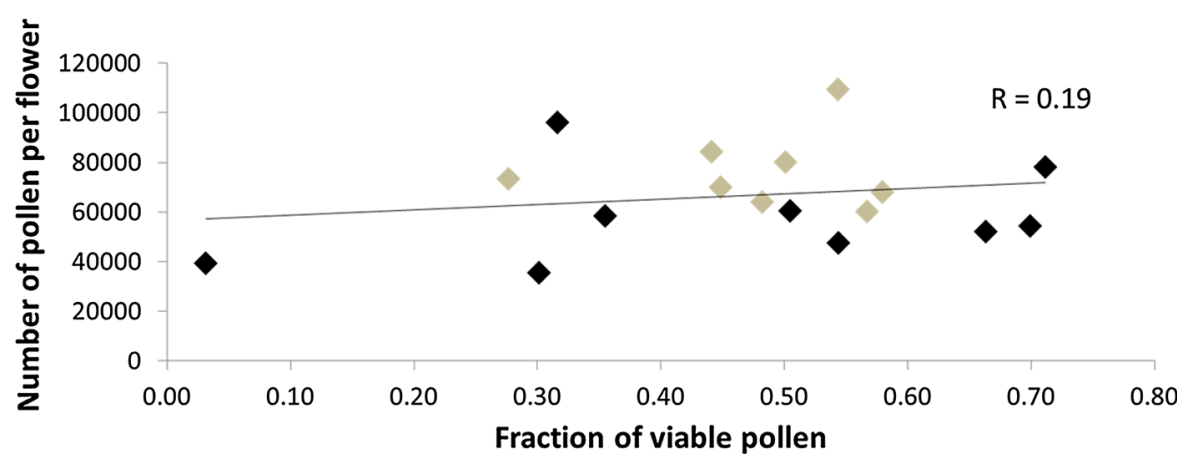

Fig. 4 Correlation between the number of pollen per flower and the fraction of viable pollen produced under heat stress by different tomato genotypes tested. Light dots represent

This might suggest that different accessions may use different strategies to ensure a successful pollination in similar high temperature environments. This can be achieved e.g. by increasing the total number of pollen produced by a flower, by keeping the fraction of viable pollen high under high temperature, or a combination of both. Besides, although we did not assess the number of produced flowers, this trait might also be of interest for the tomato production under high temperature.

The analysis of the two parameters 'the total number of pollen produced per flower' and 'the fraction of viable pollen' indicated that different genotypes vary in their response to high temperatures. For example, accession LA1580 was among the best total pollen producers under high temperature, but did not belong to the group of genotypes with the highest fraction of viable pollen. Saladette belonged to the cluster with the highest fraction of viable pollen, but on the other hand it was one of the lowest total pollen producers. No significant correlation was found between the total number of pollen and the fraction of viable pollen. This suggests that the two parameters can represent independent pollen quality traits. Combining these traits could provide the best reproductive capability under high temperature and, likewise, the product between the total number of pollen grains per flower x pollen viability might be used as a parameter for selecting the most thermo-tolerant accessions. Solanum pimpinellifolium LA2854, CLN1621F, and Nagcarlang, seem to be potentially the most heat tolerant accessions of the 17 genotypes tested, since they showed the highest fraction of viable pollen and a high total number of pollen. The analysis of pollen genotypes grown in the first period whereas dark dots represent genotypes grown in the second period. The correlation factor was calculated based on Pearson Correlation

viability of the 17 genotypes was however performed only under high temperature, therefore we cannot exclude that under control conditions (e.g. optimal temperature for cultivated tomato) these genotypes may also show variation of these two parameters. Nevertheless, the ability to provide a good pollen quality under high temperature remains primordial to produce fruit.

Genotypes LA2854, Nagcarlang, CL5915-153D43-3-0, and CL1131-0-0-13-0-6, CLN1621F and CL5915-93D4-1-0-3 were the best genotypes regarding the fraction of viable pollen (from 0.50 to 0.71 ). A high fraction of viable pollen, in general, is correlated with a high fruit set in tomato (Firon et al. 2006). Recently, Kartikeya et al. (2012) analysed the percentage of fruit set relative to the number of total flowers and the percentage of viable pollen among 36 tomato varieties grown under high temperature and a high positive correlation between fruit set and pollen viability was observed. Based on this observation, we can speculate that the five best genotypes for the fraction of viable pollen may also have a high fruit set under high temperatures.

Indeed, some of the tested genotypes with a fraction of viable pollen higher than 0.5 , such as CLN1621F, CL5915-93D4-1-0-3 and Saladette have already been shown to be tolerant to high temperatures in other studies (Da Costa et al. 2011; Lin et al. 2010; Abdulbaki 1991). For example, CLN1621F produced a high number of fruits per plant under high temperature, but these fruits generally had a small fruit weight (Da Costa et al. 2011). Tolerance to high temperature has indeed been shown to negatively correlate with fruit size (Wessel-Beaver and Scott 
1992). The line CL5915-93D4-1-0-3 has already been used as a heat tolerant parent in a mapping population to identify quantitative trait loci (QTL) related to fruit quality, seed set and Brix under high temperature (Lin et al. 2010). Additionally, our results confirmed that M-82 is sensitive to high temperature with a fraction of pollen viability of 0.27 . These results are consistent with those of Mazzeo et al. (2010). Although the environmental conditions of the two screening experiments we performed were kept identical, they were performed during two different adjacent time periods. Therefore, we cannot exclude that the growing period could have an effect on the total pollen production and pollen viability. Nevertheless, this study allowed us to discriminate between genotypes with a high pollen viability and genotypes with a low pollen viability.

Pollen development is one of the most sensitive processes in plants (Dane et al. 1991) and pollen quality is a major determinant of fruit production under high temperature. Several mechanistic studies have been conducted to get more insight in pollen thermo-tolerance mechanisms. These studies focussed on different aspects of pollen development, such as pollen germination (Firon et al. 2006), thermo-sensitivity of pollen developmental stages (Sato et al. 2002), as well as monitoring temperature-induced changes in transcriptomic and proteomic profiles [Frank et al. 2009; Jagadish et al. 2010 (in anthers)]. The identification of tomato genotypes with high pollen viability under high temperature conditions not only forms a valuable resource to study pollen thermotolerance mechanisms, but also helps to understand the underlying genetics and to breed for thermo-tolerance. Recently, a QTL study of pollen viability under high temperature has been carried out using a mapping population derived from CLN 1621L (tolerant) and CA4 (sensitive) tomato genotypes (Kardivel 2010). In this study, QTL LOD scores for pollen viability were low, suggesting a high complexity of this trait, where, besides genetic, many other factors may play a role. This is in line with the low heritability of tolerance to high temperature observed previously in tomato from a cross of CL5915-93D4-1-0-3 (tolerant) with UC204A (sensitive) (Hanson et al. 2002) and from cross combinations between CLN 2413R, CLN 2116B and COML CR-7 (tolerant) with the cultivars Patharkuchi and Ratan (sensitive) (Hazra et al. 2009). In agreement with the fact that other environmental factors than high temperature may play a role in the tolerance to high temperature, we noticed that the viable pollen fraction of tomato plants under high temperature may be variable regarding the time of the year in which the plants were grown before their transfer to the climate chamber (data not shown). These observations underlined the fact that other environmental factors influence the effect of high temperature stress on pollen viability.

Unravelling the genetics of a complex trait like pollen thermo-tolerance requires good insight in the mechanisms underlying pollen thermo-tolerance, in order to dissect this complex trait into sub-traits with simpler genetics. Furthermore, the success of such genetic studies is dependent on the availability of well characterised plant materials displaying variation in different parameters contributing to thermo-tolerance. Therefore, every new accession tolerant to high temperatures deserves to be studied for thermotolerance in detail. In this study, new thermo-tolerant (LA2854, LA1478 and LA0417) as well as thermosensitive (LA1719, LA1580, and SWEET4) genotypes have been identified. These can be used as novel genetic resources to get more insight into pollen thermo-tolerance mechanisms.

Acknowledgements Marine J. Paupière, Ivo Rieu and Arnaud G. Bovy were supported by the Solanaceae Pollen Thermotolerance-Initial Training Network (SPOT-ITN; Grant Agreement 289220). Ivo Rieu received funding from TTI Green Genetics (Project 4CFD047RP). We thank Andre Maassen and Gerrit Stunnenberg (Unifarm, Wageningen UR) for the excellent care of the plants.

\section{Compliance with ethical standards}

Conflicts of interest The authors declare no conflict of interest.

Open Access This article is distributed under the terms of the Creative Commons Attribution 4.0 International License (http:// creativecommons.org/licenses/by/4.0/), which permits unrestricted use, distribution, and reproduction in any medium, provided you give appropriate credit to the original author(s) and the source, provide a link to the Creative Commons license, and indicate if changes were made.

\section{References}

Abdelmageed AHA, Gruda N (2009) Influence of high temperatures on gas exchange rate and growth of eight tomato cultivars under controlled heat stress conditions. Eur J Hortic Sci 74(4):152-159 
Abdulbaki AA (1991) Tolerance of tomato cultivars and selected germplasm to heat-stress. J Am Soc Hortic Sci 116(6):1113-1116

Alexander MP (1980) A versatile stain for pollen fungi, yeast and bacteria. Stain Technol 55(1):13-18

Da Costa CA, Da Silva AC, Sampaio RA, Martins ER (2011) Productivity of determinate growth tomato lines tolerant to heat under the organic system. Hortic Bras 29(4):590-593

Dane F, Hunter AG, Chambliss OL (1991) Fruit set, pollen fertility, and combining ability of selected tomato genotypes under high temperature field conditions. J Am Soc Hortic Sci 116(906-910):906-910

Firon N, Shaked R, Peet MM, Pharr DM, Zamski E, Rosenfeld K, Althan L, Pressman E (2006) Pollen grains of heat tolerant tomato cultivars retain higher carbohydrate concentration under heat stress conditions. Sci Hortic 109(3):212-217. doi:10.1016/j.scienta.2006.03.007

Frank G, Pressman E, Ophir R, Althan L, Shaked R, Freedman M, Shen S, Firon N (2009) Transcriptional profiling of maturing tomato (Solanum lycopersicum L.) microspores reveals the involvement of heat shock proteins, ROS scavengers, hormones, and sugars in the heat stress response. J Exp Bot 60(13):3891-3908. doi:10.1093/jxb/ erp234

Hanson PM, Chen J-T, Kuo G (2002) Gene action and heritability of high-temperature fruit set in tomato line CL5915. HortScience 37:172-175

Hazra P, Ansary SH, Dutta AK, Balacheva E, Atanassova B (2009) Breeding tomato tolerant to high temperature stress. Acta Hort 830:241-248

Hurd RG, Cooper AJ (1970) The effect of early low temperature treatment on the yield of single inflorescence tomatoes. J Hortic Sci 45:19-27

IPCC (2012) Managing the risks of extreme events and disasters to advance climate change adaptation. In: Special report of the intergovernmental panel on climate change. Cambridge university press, Cambridge

Jagadish SV, Muthurajan R, Oane R, Wheeler TR, Heuer S, Bennett J, Craufurd PQ (2010) Physiological and proteomic approaches to address heat tolerance during anthesis in rice (Oryza sativa L.). J Exp Bot 61(1):143-156. doi: $10.1093 /$ jxb/erp289

Kardivel P (2010) Molecular breeding in vegetable cropschallenges and opportunities. Paper presented at the 2nd national workshop on marker-assisted selection for crop improvement Patancheru, AP
Kartikeya S, Sunil K, Surender K, Pravin P, Vaishampayan A (2012) Screening of tomato genotypes for reproductive characters under high temperature stress conditions. Sabrao J Breed Genet 44(2):263-276

Kugblenu YO, Oppong Danso E, Ofori K, Andersen MN, Abenney-Mickson S, Sabi EB, Plauborg FL, Abekoe MK, Jørgensen ST, Jensen CR, Ofosu-Anim J (2013) Heat tolerance in field grown tomatoes (Lycopersicon esculentum Mill.) under semi-arid conditions of West Africa. Acta Hort 971:99-106

Labate JA, Robertson LD (2012) Evidence of cryptic introgression in tomato (Solanum lycopersicum L.) based on wild tomato species alleles. BMC Plant Biol 12:133. doi:10.1186/1471-2229-12-133

Lin K-H, Yeh W-L, Chen H-M, Lo H-F (2010) Quantitative trait loci influencing fruit-related characteristics of tomato grown in high-temperature conditions. Euphytica 174(1):119-135. doi:10.1007/s10681-010-0147-6

Mazzeo MF, Cacace G, Massarelli I, Leone A, Grillo S, Siciliano RA (2010) Proteomics for the elucidation of heat stress response mechanisms in anthers of tomato plants. Paper presented at the proceedings of the 54th Italian society of agricultural genetics annual congress, Matera

Peterson R, Slovin JP, Chen C (2010) A simplified method for differential staining of aborted and non-aborted pollen grains. Plant biol 1(2):66-69

Pressman E, Peet MM, Pharr DM (2002) The effect of heat stress on tomato pollen characteristics is associated with changes in carbohydrates concentration in the developing anthers. Ann Bot 2002:631-636

Sato S, Peet MM, Thomas JF (2002) Determining critical preand post-anthesis periods and physiological processes in Lycopersicon esculentum Mill. exposed to moderatly elevated temperatures. J Exp Bot 53:1187-1195

Viquez-Zamora M, Vosman B, van de Geest H, Bovy A, Visser RG, Finkers R, van Heusden AW (2013) Tomato breeding in the genomics era: insights from a SNP array. BMC Genom 14:354. doi:10.1186/1471-2164-14-354

Wahid A, Gelani S, Ashraf M, Foolad M (2007) Heat tolerance in plants: an overview. Environ Exp Bot 61(3):199-223. doi:10.1016/j.envexpbot.2007.05.011

Wessel-Beaver L, Scott JW (1992) Genetic variability of fruit set, fruit weight, and yield in a tomato population grown in two high-temperature environments. J Am Soc Hortic Sci 117(5):867-870 